\title{
Curcumin and Its Biological Importance: A Review
}

\author{
Praveen Kumar Dikkala $^{1 *}$ and S.D.S.N. Shirisha ${ }^{2}$ \\ ${ }^{1}$ Professor Jayashankar Telangana State Agricultural, University, Hyderabad-500030, \\ Telangana, India \\ ${ }^{2}$ APAC, LAM farm, Andhra Pradesh Agricultural University, Guntur, Andhra Pradesh, India \\ *Corresponding author
}

\begin{tabular}{|l|}
\hline Ke y w o r d s \\
Curcumin and \\
Biological \\
Importance, \\
Anti-inflammatory \\
\hline Article Info \\
\hline $\begin{array}{l}\text { Accepted: } \\
\text { 10 January } 2018 \\
\text { Available Online: } \\
\text { 10 February } 2018\end{array}$ \\
\hline
\end{tabular}

\section{Keywords}

Curcumin and

Biological

Importance,

Article Info

Accepted:

Available Online.

10 February 2018

\section{A B S T R A C T}

Turmeric is a spice that has been used extensively used in the different food sectors as a flavoring and coloring agent, in almost every cellular biological event, process includes in the gene expression, signaling and regulation. The bioactive polyphenol present in the turmeric is curcumin, which plays an important role in the anti-inflammatory, anti-oxidant, anticarcinogenic, anti-invasive as a mediator of chemo resistance, radio resistance, chemopreventive agent, and as a therapeutic agent in wound healing, dyspepsia,diabetes and cardiovascular ailments, rheumatism, body ache, hepatic disorders, skin diseases, intestinal worms, constipation, diarrhoea, intermittent fevers, urinarydischarges, inflammations, biliousness, leukoderma, amenorrhea, and colic. Curcumin has the potential to treat a wide variety of inflammatory diseases including cancer, diabetes, cardiovascular diseases, arthritis, Alzheimer's disease andpsoriasis through modulation of numerous molecular targets. With time more and more of its medicinal uses were discovered and today curcumin is associated with a plethora of beneficial effects on human health.

\section{Introduction}

Now a day's many number of plant derivatives such as flavonoids and polyphenols, have gained much attention due to their specific biological effects. In these different compounds, curcumin has emerged as a bioactive compound with multiple biological properties. Curcumin is a phenolic compound derived from turmeric. Turmeric is a spice that has been used extensively in different food items in India as a flavoring and coloring agent. The use of turmeric as a medicinal compound long back from 2000 $\mathrm{BC}$ which is used as an anti-inflammatory agent. With time more and more of its medicinal uses were discovered and today curcumin is associated with a plethora of 
beneficial effects on human health. So much of research in last decade has led to identify the various sites and mechanisms of action of curcumin and that studies concluded that an effect of curcumin in almost every cellular biological event, process includes in the gene expression, signaling and regulation. Curcumin in the form of 1,7-bis(4-hydroxy-3methoxy phenyl) -1,6-heptadiene-3,5-dione) acts as a phenolic compound and is the major ingredient in the rhizome of the herb curcuma longa, which is extracted as a yellow pigment from the powdered form which is called as turmeric, the rhizome. Today, curcumin finds use as an anti-inflammatory, anti-mutagenic (Stoner and Mukhtar., 1995) and anti-cancer molecule (Kuttan et al., 1985). It works as an anti-oxidant and is capable of inducing apoptosis (Kuo et al., 1996 and Khar et al., 1999). Curcumin is a polyphenolic, diferuloylmethane compound responsible for the yellow pigmentation (Goel et al., 2008). It has been shown clearly that curcumin acts as an antiproliferative, antiangiogenic agent and anti-invasive as a mediator of chemoresistance and radioresistance as a chemopreventive agent, and as a therapeutic agent in wound healing, diabetes and cardiovascular ailments as indicated by over 6,000 citations (Sharma et al., 2005)

In addition to this many clinical studies have been carried out with curcumin. One of the major problems with curcumin is perceived with the bioavailability. Curcumin should be delivered in vivo, how it is bioavailable, how well curcumin is absorbed and how it is metabolized, is the focus of this review. Various formulations of curcumin that are currently available are also discussed in this paper

\section{Biological importance of curcumin}

Turmeric powder has many biological activities which increases the mucin secretion thus it act as a gastric protection. Curcumin has many beneficial effects on the intestine.

Curcumin decreases the density of lipoprotein which significantly affects the plasma and total cholesterol level in liver along with an increase of alpha-tocopherol level. This clearly shows that the in vivo interaction between curcumin and alpha tocopherol may increase the bioavailability of vitamin $\mathrm{E}$ and decrease the cholesterol level. Curcumin binds with egg and soy-phosphatidylcholine, which in turn binds divalent metal ions that makes the antioxidant activity. Induced liver damage is significantly decreased by curcumin treatment and the arachidonic acid level is increased with increase in curcumin intake (Bagchi, 2012).

Curcumin also shows the protection aganist the ulcerogenic effects of phenylbutazone in guinea pigs at a dose of $50 \mathrm{mg} / \mathrm{kg}$. Antiflatulent activity of curcumin was observed in both in vivo and in vitro experiments in rats. Sodium curcuminate from turmeric is acts as a antispasmodic activity which is observed in guinea pig ileum.

Turmeric accelerates the wound healing process and it has great potential for wound healing. The use of turmeric at the site of an injury by topical application promotes healing of wounds. Therefore the turmeric can be used in different forms for treating different ailments including surgical wounds (Parveen et al., 2017).

Curcumin have the enhancing capacity of intestinal lipase, sucrase and maltase activity.it also has the capacity to reduce the pathological effect and it protects from the damage caused by myocardial infarction. Curcumin increased the $\mathrm{Ca}^{2+}$ transport and its slippage from the cardiac muscle sarcoplasmic reticulum, thereby raising the 
possibility of medicinal interventions to correct the defective $\mathrm{Ca}^{2+}$ homeostasis in the cardiac muscle. It was observed that curcumin has significant reduction of cholesterol in the hypercholesteremic rats. The antioxidant activity present in curcumin reduced the vascular dementia on nervous system by curcumin and its manganese complex.

Turmeric oleoresin effected the change in gene expression were observed in mice and they are considered to be the mechanism by which the turmeric oleoresin affected the control of both blood glucose levels and abdominal adipose tissue masses. All of these results show that the use of whole turmeric oleoresin is more effective than the use of either curcuminoids or the essential oil alone (Honda et al., 2006)

\section{Biological activities of curcumin}

\section{Hypoglycemic effect}

Curcumin and its analogs have been synthesized to improve its hypoglycemic efficacy which helps for the diabetic people. For an instance, a novel curcumin derivative (NCD) was developed through the covalent modification of the curcumin molecule on sites remote from its natural functional groups.

This novel curcumin derivative (NCD) was tested on the diabetic rats to determine whether it exhibits a hypoglycemic effect. The results clearly showed that it lowered the plasma glucose by 27.5 percent and increased plasma insulin by 66.67 percent (Abdel Aziz et al., 2012).

Later, it was observed that this novel curcumin derivative was partially mediated by induction of the HO-1 gene. Another curcuminoid derivative, bis methane, inhibited these levels of plasma glucose in the diabetic rats. This is clearly evident that curcumin derivatives exhibit antidiabetic activities.

\section{Anticancer effects}

Curcumin has shown to display chemotherapeutic as well as the chemo preventive effects in different types of cancers. Mono carbonyl analog of curcumin is synthesized from several chemical modifications in the basic structure of the curcumin is to increase its biological activity and also the bioavailability of curcumin. In vitro assays showed that this curcumin derivatives had greater antiproliferative effects on colon cancer cells than curcumin (Zheng et al., 2013). Another curcumin derivative such as, hydrazine benzoyl curcumin, induced A549 cell autophagy and inhibited the viability of A549 cells to $76.68 \%$ after 24 hours of treatment (Zhou et al., 2014). Autophagy is elicited by bDHC before cell death. The curcumin derivative bis-DeHydroxy curcumin also has been shown to induce autophagy on human colon cancer cells, but not on human normal cells (Basile et al., 2013).

High cost, toxicity and less success rate and patient incompliance associated with existing anticancer drugs necessitated for finding new anticancer drugs which can be overcome the aforementioned drawbacks. Toward this direction effort has been made to understand the anticancer activity of curcumin in combination with genistein reduced the human prostate cancer cell line cells with respect to their antiangiogenic effect. Curcumin in combination with genistein had showed the time dependent decrease in cell viability, increase in apoptosis and cell cycle arrest at $\mathrm{G}_{0}$ phase. These effects were more noticeable when curcumin is used very less in combination with genistein. To understand antiangiogenic effect of this combination, 
expression of ARNT and HIF-1 $\alpha$ was studied. Significant decline in expression of ARNT and HIF-1 $\alpha$ protein level was seen in comparison to control group and their respective monotherapy treated groups. Curcumin and genistein are very effective in abrogating the VEGF production by evading ARNT and HIF-1acomplex formation as proved by immunoprecipitation assay. Thus curcumin showed that it is very effective (Aditya et al., 2014)

Several studies clearly showed that curcumin has potential against several cancers including leukemia, lymphoma, melanoma, and sarcoma, as well as gastrointestinal, genitourinary, breast, ovarian, head and neck, lung, and neurological cancers (Anand et al., 2008). Curcumin acts at different stages of cancer development. It blocks transformation, tumor initiation, tumor promotion, invasion, angiogenesis, and metastasis. In vitro and animal studies have revealed that curcumin suppresses carcinogenesis and inhibits the proliferation of a wide variety of tumor cells (Aggarwal et al., 2003). Curcumin decreases the growth of tumor cells through regulation of multiple cell signaling pathways such as cell survival pathway, cell proliferation pathway, caspase activation pathway, tumor suppressor pathway mitochondrial pathways, death receptor pathway, and protein kinase pathway to effect the tumor growth (Ravindran et al., 2009).

\section{Anti-inflammatory effects}

A lipophilic derivative and hydrophilic derivatives of curcumin such as diacetyl curcumin and diglutaryl curcumin showed in vivo to have an analgesic and antiinflammatory activities. A carrageenan induced paw edema model indicated antiinflammatory activity to all curcumin derivatives. The percentage inhibition in the paw edema was higher in diacetyle curcumin than in curcumin (Jacob et al., 2013). Pan et al., (2013) reported that curcumin analog B06 showed an enhanced anti-inflammatory activity compared with that of curcumin through inhibition of c-Jun N-terminal kinase/NF- $\kappa \mathrm{B}$ activation. In vivo, curcumin derivative (B06) treated animals showed a significant decrease in inflammatory mediators in the serum and kidneys and in heart and renal macrophage infiltration. Another analog, 2,6-bis-4- cyclohexanone, or BHMC, inhibited the synthesis of several inflammatory mediators. Thus, curcumin analogs play a potential role to inhibit inflammatory factors.

\section{Antioxidant effects}

Synthetic sugar derivative in the curcumin has more powerful antioxidant properties. Curcumin decreases the amyloid- $\beta$ and tau peptide aggregation at micromolar concentrations, whereas the sugar-curcumin conjugate inhibits this aggregation at concentrations as low as the nanomolar level. 5-chloro curcumin which is obtained from natural curcumin, has free radical scavenging activity. CNB-001, a pyrazole derivative of curcumin, protects neuronal cells against toxicity by decreasing free radical formation, and reduces apoptosis by its action on mitochondria. Semi carbazone derivative of curcumin has also shown efficient antioxidant and antiproliferative activity, although its antiradical activity was less than that of curcumin. The probable site of attack for CRSC is both the $\mathrm{OH}$ phenolic group and the imine carbonyl position (Dutta et al., 2005).

\section{In vitro and In vivo study on curcumin}

Many studies on curcumin revealed that various cancer cells on the various parts on body lines have demonstrated the decreased in cell growth and survival, concomitant with the compound's effects on molecular 
pathways involved in the cellular proliferation. Expression of constitutively active $\mathrm{NF}-\kappa \mathrm{B}$ and $\mathrm{I} \kappa \mathrm{K}$ has been observed in multiple oral squamous cell carcinoma cell lines, and curcumin treatment was shown to suppress growth and survival of these cell lines via inhibition of NF- $\kappa \mathrm{B}$ activation (Chun et al., 2003). Signal transducer and activator of transcription 3 is a signaling protein observed to be overexpressed in multiple head and neck cancers, and curcumin was shown to suppress the IL-6 mediated phosphorylation of Signal transducer and activator of transcription 3 as well as inhibiting nuclear localization (Chakravarti et al., 2006). Curcumin has demonstrated in vivo growth suppressive effects on head and neck squamous cell carcinoma using nude mouse xenograft models. The lipophilic nature of curcumin and relative insolubility in aqueous solutions, combined with short half-life and low bioavailability following oral administration has presented a significant challenge in developing an effective delivery system for its use as a chemotherapeutic agent (Bisht and Maitra, 2009). In an effort to overcome this obstacle, various strategies are being tried including the use of piperine as an adjuvant agent to slow curcumin breakdown as well as the development of liposomal, phospholipid and nano-particulated formulations of the compound to enable intravenous administration (Bisht and Maitra, 2009). Liposomal formulations of curcumin have been studied in various cancers including pancreatic, colorectal and prostate (Li et al., 2005; Li et al., 2007; Mach et al., 2009).

\section{References}

Abdel Aziz, M.T., El-Asmar, M.F., El-Ibrashy, I.N., Rezq, A.M., Al-Malki, A.L., Wassef, M.A., et al., 2012. Effect of novel water soluble curcumin derivative on experimental type-1 diabetes mellitus (short term study). Diabetology \& Metaboilc syndrome. 4:30.

Aditya, N.P., Shim, M., Yang, H., Lee, J.Y., Ko S. 2014. Antiangiogenic effect of combined treatment with curcumin and genistein on human prostate cancer cell line. Journal of Functional Foods. 8: 204-213

Aggarwal, B.B., Kumar, A., Bharti, A.C. 2003. Anticancer potential of curcumin: preclinical and clinical studies. Anticancer Research 23:363-98.

Anand, P., Sundaram, C., Jhurani, S., Kunnumakkara, A.B., Aggarwal, B.B. 2008. Curcumin and cancer: an "old-age" disease with an "age-old" solution. Cancer Letters 267:133-64

Bagchi, A.2012. Extraction of Curcumin. IOSR Journal of Environmental Science Toxicology Food Technology. 1:1-16.

Basile, V., Belluti, S., Ferrari, E., Gozzoli, C., Ganassi, S., Quaglino, D., et al., 2013. bisDehydroxy curcumin triggers mitochondrial-associated cell death in human colon cancer cells through ER-stress induced autophagy. PLoS One 8:53664.

Bisht, S., Maitra, A.2009. Systemic delivery of curcumin: 21st century solutions for an ancient conundrum. Current Drug Discoverey Technology. 6:192-199.

Chakravarti, N., Myers, J.N., Aggarwal, B.B. 2006. Targeting constitutive and interleukin-6-inducible signal transducers and activators of transcription 3 pathway in head and neck squamous cell carcinoma cells by curcumin (diferuloylmethane). International Journal of Cancer.119:12681275 .

Chun, K.S., Keum, Y.S., Han, S.S., Song, Y.S., Kim, S.H., Surh, Y.J.2003. Curcumin inhibits phorbol ester-induced expression of cyclooxygenase-2 in mouse skin through suppression of extracellular signalregulated kinase activity and NF-kappaB activation. Carcinogenesis. 24:1515-1524.

Dutta, S., Padhye, S., Priyadarsini, K.I., Newton, C. 2005. Antioxidant and antiproliferative activity of curcumin semicarbazone. Bioorganic \& Medicinal Chemistry Letters 15:2738-44.

Goel, A.B., Kunnumakkara, B.B.2008. Aggarwal. Curcumin as "Curecumin": from kitchen to 
clinic. Biochemical Pharmacology. 75:787809.

Honda, S., Aoki, F., Tanaka, H., Kishida, H., Nishiyama, T., Okada, S., Matsumoto, I., Abe, K., Mae, T.2006. Effects of ingested turmeric oleoresin on glucose and lipid metabolisms in obese diabetic mice: a DNA microarray study. Journal of Agriculture and Food Chemistry. 54(24):9055-62.

Jacob, J.N., Badyal, D.K., Bala, S., Toloue, M.2013. Evaluation of the in vivo antiinflammatory and analgesic and in vitro anti-cancer activities of curcumin and its derivatives. Natural Product Communications. 8:359-62.

Khar, A., Ali, M., Pardhasaradhi, B.V.V., Begum, Z., Anjum, R. 1999. Antitumor activity of curcumin is mediated through the induction of apoptosis in AK-5 tumor cells. FEBS Letters. 445-165

Kuo, M.L., Huang, T.S., Lin, J.K.1996. Curcumin, an antioxidant and anti-tumor promoter, induces apoptosis in human leukemia cells. Biochimica et Biophysica Acta (BBA). 1317-95.

Kuttan, R., Bhanumathy, P., Nirmala, K., George, M.C.1985. Potential anticancer activity of turmeric (Curcuma longa). Cancer Letters. 29:197.

Li, L., Ahmed, B., Mehta, K., Kurzrock, R. 2007. Liposomal curcumin with and without oxaliplatin: effects on cell growth, apoptosis, and angiogenesis in colorectal cancer. Molecular Cancer Therapeutics. 6:1276-1282.

Li, L., Braiteh, F.S., Kurzrock, R. 2005. Liposome-encapsulated curcumin: in vitro and in vivo effects on proliferation, apoptosis, signaling, and angiogenesis. Cancer. 104:1322-1331.

Mach, C.M., Mathew, L., Mosley, S.A., Kurzrock, R., Smith, J.A. 2009. Determination of minimum effective dose and optimal dosing schedule for liposomal curcumin in a xenograft human pancreatic cancer model. Anticancer Research.29:1895-1899.

Parveen, A.L., Syed, W.A., Vivek P., Bashir A. 2017. Role of turmeric in management of alveolar osteitis (dry socket): A randomised clinical study. Journal of Oral Biology and Craniofacial

Researchhttp://dx.doi.org/10.1016/j.jobcr.2 017.08.005

Ravindran, J., Prasad, S., Aggarwal, B.B.2009. Curcumin and cancer cells: how many ways can curry kill tumor cells selectively. AAPS Journal. 11:495-510.

Sharma R.A., Gescher A.J., Steward W.P.2005. Curcumin: the story so far. European Journal of Cancer. 41:1955-1968

Stoner, G.D., Mukhtar, H. 1995. Polyphenols as cancer chemopreventive agents. Journal of Cellular Biochemistry. Supplement. 22:169-80.

Zheng, A., Li, H.,Wang, X., Feng, Z., Xu, J., Cao, $\mathrm{K}$, et al., 2014. Anticancer effect of a curcumin derivative B63: ROS production and mitochondrial dysfunction. Current Cancer Drug Targets. 14(2):156-66.

Zhou, G.Z., Zhang, S.N., Zhang, L., Sun, G.C., Chen, X.B. 2014. A synthetic curcumin derivative hydrazine benzoylcurcumin induces autophagy in A549 lung cancer cells. Pharmaceutical Biology. 52:111-6.

\section{How to cite this article:}

Praveen Kumar Dikkala and Shirisha, S.D.S.N. 2018. Curcumin and Its Biological Importance: A Review. Int.J.Curr.Microbiol.App.Sci. 7(02): 1100-1105. doi: https://doi.org/10.20546/ijcmas.2018.702.137 